\title{
Development of methodology for analysis of the FADS1 genetic variants - first pilot study in Bulgaria
}

\author{
Elena Kuzova, \\ Tzveta Georgieva, \\ Vesselka Duleva \\ National Center of Public Health and Analyses, \\ Acad. Ivan Evst. Geshov 15 blvd, 1431 Sofia, \\ Bulgaria \\ Received: $20^{\text {th }}$ September 2016 . \\ Corresponding author: \\ Elena Kuzova \\ Address: National Center of Public Health and \\ Analyses, Acad. Ivan Evst. Geshov 15 blvd, \\ 1431 Sofia, Bulgaria, GMO Unit \\ E-mail address: elenakuzova@gmail.com \\ Mobile: +359883497351, +35928056361
}

\begin{abstract}
Introduction: A particular single nucleotide polymorphism (rs174547) was identified in the FADS1 gene according to published data, with significant association between its variants and the metabolism of fatty acids.

Objective: To develop, adapt and verify methodology for detection and analysis of FADS1 genetic variants (SNPS) in a small scale setting (pilot study), as these SNPs have been associated with the metabolism of fatty acids.

Methods: A specific primer set targeted at the SNP region was designed and the reaction was optimized under various conditions: primer concentrations, reaction temperatures, duration and number of amplification cycles, etc.

A pilot group of 10 subjects from Bulgarian volunteers were randomly picked and tested in order that the optimized conditions were verified. Following standard DNA extraction from buccal swabs, a polymerase chain reaction was performed amplifying a 243 bp fragment, with the $C / T$ SNP contained inside. The amplicon was visualized after 40 min electrophoresis on a $2 \%$ agar's gel. Products were then run on a capillary sequencer (Sanger type sequencing), and obtained data were read with the MEGA5 software.

Results: The proposed system for detection and analysis of SNPS in the FADS1 gene was optimized and probated for the first time in Bulgaria. Thus, although a small-scale pilot study, its results represent primary data originated from Bulgarian individuals. It was shown that under the optimized reaction condition our primer systems produced an amplicon with the expected size and indeed contained a fragment from the FADS1 gene, with the analyzed C/T SNP inside.

Conclusions: The introduction of molecular biomarkers would allow an individual assessment of the fatty acid metabolism to be made.Thereby, the developed and probated methodology, if is to be applied in a large scale setting, would help to investigate the relationship and influence of genetic predisposition, individual metabolism and food intake.
\end{abstract}

Keywords: genetic variants, FADS1 genes, metabolism of fatty acids, personalized nutrition

\section{INTRODUCTION}

Fatty acid desaturases (FADS) are membranebound key enzymes in the endogenous conversion of a-linolenic acid (ALA, C18:3n-3) and linoleic acid (LA, C18:2 n-6) into long-chain PUFAs (Poly Unsaturated Fatty Acids). D5D stands for delta-5 desaturase and D6D is delta-6 desaturase. These key enzymes are encoded by gene stermed FADS1 and FADS2 which are located in human chromosome 11 (11q12q13.1) separated by an 11 $\mathrm{kb}$ region Figure 1 (adaptation according to Linda Schaeffer et al $(1)(2,3)$. There is also aFADS3 gene situated in the same cluster, but the function of its translated product remains unknown (4). D5D and D6D are the rate limiting enzymes in the synthesis of long-chain PUFAs, arachidonic acid (AA), eicosapentaenoic acid (EPA) and docosahexaenoic acid (DHA) from their dietary precursors LA and ALA. On the other hand AA and EPA are the precursor fatty acids for formation of eicosanoids and DHA for transformation into docosanoids.

D6D catalyzes the metabolic reaction of conversion of a-linolenic acid (ALA, 18:3n-3) and linoleic acid (LA, 18:2n-6) into stearidonic acid (STD, 18:4n-3) and $\mathrm{y}$-linolenic acid (GLA, 18:3n6), respectively, Figure $\mathbf{2}$ (adapted to Lin Xie et al) (5). Then an elongation step is performed, after which D5D introduces a double bond at the $\Delta 5$ position in the 20 -carbon fatty acid chain. D5D catalyzes the conversion of eicosatetraenoic acid (ETA, 20:4n-3) and dihomo-y-linolenic acid (DGLA, 20:3n-6) into eicosapentaenoic acid (EPA, 20:5n$3)$ and arachidonic acid $(A A, 20: 4 n-6)$, respectively $(6,7)(2)$, Figure 2 .

Figure 2. Desaturation and elongation of $n-3$ and $n-6$ fatty acids

Above mentioned PUFAs serve as important 
precursors for lipid-derived modulators of cell signaling, gene expression and inflammatory processes (8). Irregularities in membrane fatty acid compositions have been associated with several chronic non-communicable human diseases such as autoimmune disorders (9), coronary heart disease (10), cancer (8), atopic eczema (11) and Alzheimer's disease (12). Furthermore, D5D and D6D participate in the major metabolic pathway for synthesis of pro-inflammatory and antiinflammatory eicosanoids. Eicosanoids produce a wide range of biological effects on inflammatory responses (predominantly those of the joints, skin and eyes), on the intensity and duration of pain and fever, and on reproductive function (including the induction of labor) (13)(14). They also play important roles for inhibition of gastric acid secretion, regulation of blood pressure through vasodilatation or constriction, and inhibition or activation of platelet aggregation and thrombosis. The principal eicosanoids of biological significance to humans are a group of molecules derived from the C20 fatty acid, arachidonic acid. Additional biologically significant eicosanoids are derived from dihomo- $y$-linolenic acid which is produced in the reaction pathway transforming linoleic acid to arachidonic acid, Figure 2. Minor eicosanoids are derived from eicosapentaenoic acid which is itself

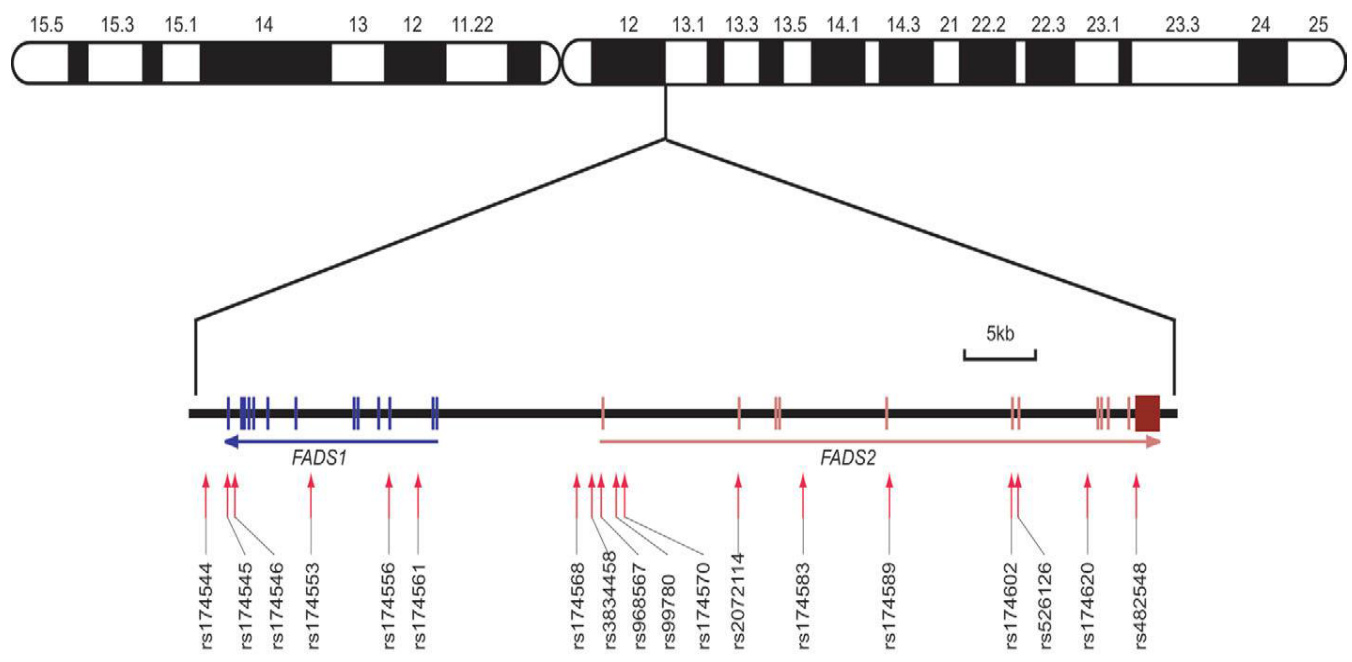

Figure 1. Structure of FADS1 and FADS2 gene cluster, its localization on human chromosome 11 and the location of some SNPs in FADS1 and FADS2 genes. (adaptation according to Linda Schaefferet. al.-Human Molecular Genetics, 2006, Vol. 15, No. 11 1745-1756doi:10.1093/hmg/ddl117)

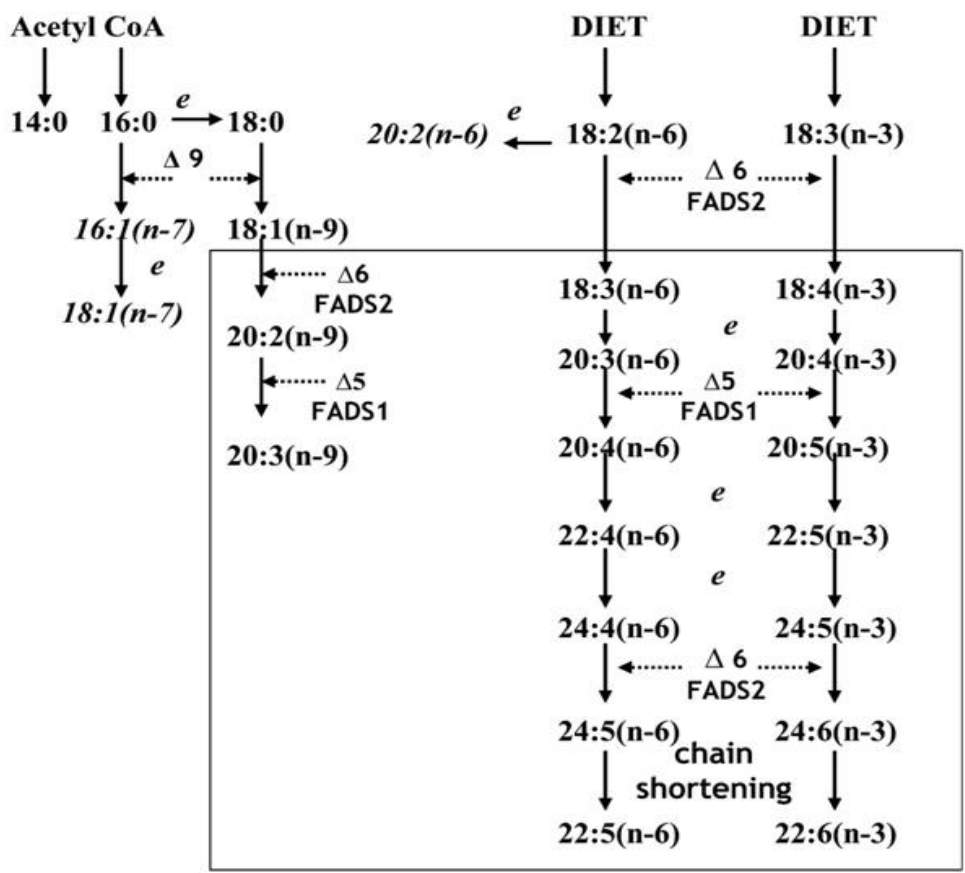

Figure 2.Desaturation and elongation of $n-3$ and $n-6$ fatty acids. The enzymesdelta- 6 desaturase and delta-5 desaturases are encoded by FADS2 and FADS1, respectively (adapted to Lin Xie, and Sheila M. Innis J. Nutr. 2008;138:2222-2228) 
derived from a-linolenic acid or obtained in the diet.

The FADS1 and FADS2 gene cluster involved in the metabolic pathway of LA and ALA are polymorphic. Recent studies on the polymorphism therein indicate that minor alleles of the genetic variants in FADS1and FADS2 are associated with higher levels of LA and lower AA levels in red blood cell membrane and plasma phospholipids that may influence the estimation of dietary requirements for prevention and for treatment purposes.

Studies on genetic variants and diet are called nutrigenetics, defined as the inherited basis for individual differences to nutrient (diet) response, whereas studies on the role of nutrients in gene expression are called nutrigenomics (15).

Some studies in the nutrigenetics field have shown that blood concentrations of LDLcholesterol, HDL-cholesterol and triglycerides(TG) have a strong genetic influence (16)(17). Single nucleotide polymorphisms (SNPs) of the FADS gene cluster that includes FADS1, FADS2 and FADS3 genes, are associated with LDL, HDL, TG (18)(19) (20)and even with levels of fasting blood glucose concentrations (20)(21). A relationship between these blood markers and the FADS1 polymorphism rs174547 (T/C) has been demonstrated in several studies $(19,20)$. Moreover, this association between genetic variations in FADS1 and LDL could be modified by the intake of dietary long-chain PUFAs (22). Hellstrand et al. observed significant interaction between variants of rs174547 and long-chain $\omega-3$ PUFA intakes on LDL $(P=0.01)$; they report that the $\mathrm{C}$-allele was only associated with lower LDL among individuals in the lowest tertile of long-chain $\omega-3$ PUFA intakes $(P<0.001)$. In addition, significant interaction was observed between variants ofrs174547 and the ratio of ALA and linoleic FA intakes on HDL $(P=0.03)$. However, no significant associations between the C-allele and HDL were determined within the intake tertiles of the ratio.

Perturbations in lipid metabolism characterize many of the chronic diseases currently affecting our modern society, such as cardiovascular diseases, type 2 diabetes, obesity, inflammation processes, alterations in the lipid status, etc. (6). Lipids have very important structural and functional biological role in living organisms. Some lipids and their derivatives are main structural component of biological membranes, such as the cellular plasma membrane and the intracellular membranes of organelles. Triglycerides, stored in adipose tissue, are a major form of energy storage both in animals and plants. In the past 10-15 years, evidence has emerged showing that lipid signaling is a vital part of the cell signaling (23)(24). Lipid signaling may occur via activation of $\mathrm{G}$ protein-coupled or nuclear receptors, and members of several different lipid categories have been identified as signaling molecules and cellular messengers. Lipids and their derivatives are also involved in membrane permeability, the process of inflammation and proliferation, immunity status and response (13, $25,26)$. Still, it remains unclear whether changes in lipid metabolism are the real cause or simply a consequence from the development of chronic diseases. However, changes in lifestyle or medical interventions in order to modify lipid levels are still the first goal to be achieved for health management and prevention.

\section{OBJECTIVE}

This study aimed to develop and verify methodology for detection and analysis of FADS1rs174547 genetic variants among the Bulgarian population. Using the proposed methodology once detected and determined the rs174547 SNP could provide valuable information concerning individual's fatty acid metabolism and may support nutritionists when nutritional requirements for omega- 3 and omega- 6 fatty acids are estimated. Second, if applied at a large scale, this methodology could give researchers a tool to obtain epidemiological data concerning the genetic distribution of the particular SNP among Bulgarian population, which is to be done for the first time in Bulgaria.

\section{METHODS}

\section{Study group}

A small-scale group of 10 Bulgaria volunteers (aged 28-65) was randomly picked for the pilot study. All volunteers were acquainted with the procedure and signed informed consent paper. A buccal swab sample was collected from the inside of the cheek and placed in 200 $\mu$ l PBS buffer for further DNA extraction.

\section{DNA extraction procedures}

DNA extraction was performed using Gene GET Genomic DNA Purification Kit (Thermo Fisher Scientific, Cat.No:K0721) from buccal swab samples, according to the manufacturer's recommendation. Quality and quantity of the extracted nucleic acid were assessed both with a spectrophotometer and an agar gel electrophoresis. Buccal swab tissue was used as a sample material mostly because of the quality of the DNA that is harvested and because of the non-invasiveness of the procedure.

\section{Primer design software}

Primer 3 designer software was used (http:// biotools.umassmed.edu/bioapps/primer3 www. cgi). The technique requires input of human partial genomic sequence, Figure $\mathbf{3}$, obtained from dbSNP genome database (http://www.ncbi.nlm.nih.gov/ snp/) by accession number of the targeted SNP (rs174547). Several parameters were determined when the primer set was generated, such as: the size of the amplicon; the content of GC; etc. When the primer set design was completed a report was generated with detailed information for each primer couple. Several sequences were chosen for synthesis and best initial results were obtained with a primer pair named FADS1-F and FADS1-R. The chosen specific primer set for the targeted SNP region, amplified a 243 bp DNA sequence. One primer consisted of 20 bp with the following sequence: CACTCCCTTCACATGGTT GC; and the other also of $20 \mathrm{bp}$, with a given sequence: GGTTCCTGGAAAGCAACTGG, Table 1. 


\section{PCR optimization process}

Several parameters such as primer concentration, reaction temperatures and times were optimised in the polymerase chain reaction (PCR). Best PCR amplification was obtained with the following protocol: $5 \mathrm{~min}$ at $95^{\circ} \mathrm{C}$, followed by 40 cycles of denaturation (30 sec at $94^{\circ} \mathrm{C}$ ); annealing ( $30 \mathrm{sec}$ at $56.5^{\circ} \mathrm{C}$ ) and extension (30 sec at $72^{\circ} \mathrm{C}$ ). All PCR reactions were run on Gene

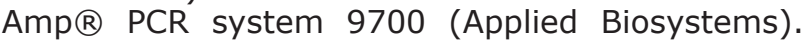
The reaction volume was $25 \mu \mathrm{l}$, and the mixture contained $12.5 \mu \mathrm{l}$ TaqMan $\AA$ Universal PCR Master Mix (cat.No4304437), $1 \mu \mathrm{l}$ of FADS1-F primer $(10 \mathrm{pmol} / \mu \mathrm{l}), 1 \mu \mathrm{l}$ of FADS1-F primer $(10 \mathrm{pmol} / \mu \mathrm{l})$, $5.5 \mu \mathrm{l}$ RNase/DNase Free water (Ferments) and $5 \mu \mathrm{l}$ diluted DNA sample. All reactions were conducted in the presence of two negative controls: 1 . RNase/
DNase Free water (Non-template control, NTC); 2. Bacterial DNA.

Amplification products with the targeted C/T SNP inside were visualized after 40 min electrophoresis on a $2 \%$ agar gel stained with ethidium bromide,

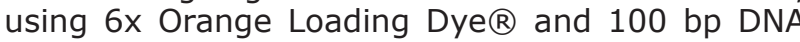
Ladder (Ferments). Following the electrophoresis, agar gels were documented with the CN-1000 Dark Room Chemiluminescence Imaging System using Bio Capt software ver.12.5.

\section{Sequencing reactions}

Last, but not least, the specificity of the obtained products was verified through Sanger sequencing (ABI3730 XL capillary sequencer). The obtained data was read with the MEGA5 software, a consensus alignment was created and $\mathrm{C} / \mathrm{T}$ polymorphism was determined for each sample, Figure 5.

1 CGGCGAAGGC TGACAGCAGG GGCTTGGACT GGTACTCTAT GCCATGCTTG GCACACAAGC

61 ACTGCACCAG GGGAGCCACT TTGTGGTAAT TGTGTCGAGG CATCGTGGGA AAAAGACTTT

121 AGGGAGAACG GGGGAGTCAG TGGTTCCTGC TTCTCTGCTC CCACCTGTAC CCAACACTTA

181 CACCCTCCCC AGGACCCTGC TTCCCCAGGC TCCCTACTCA CTGGTGCTCA ATCTGGAAGT

241 TGAGGTGTCC ACTGAACCAG TCATTGAAGG CAGACTTGTG GACATTGCAT GTGGCCTGGA

301 GCTGGCGGAA AAGGTCAGGG GAGAGCATGT TGAATATCAG ATGGAAAGGC CAGCCCAGCA

361 TTCTCCAGGT AAAGCTGGCT GAGGAAGGGA CATGAGACTG TCTTGGTCAC TCCCTTCACA

421 TGGTTGCAGA ACAAGAGCCT CAGGCTAATG AGAAAATGCT GTTTGGGGGA CTTTTTGTTT

481 TTGCTGTTTT CACCTACGCA

Y (rs174547)

502 CCTTTTCAAT AGTTGTGTTA TGCTCCAGTC TTCTGAGTGA CTGTCCCTTA CCTGGGTGGA

562 AACCCAGTCC ATGTTCCGGT CATGATCAAT GTGCATGGGA ATATGGTTCA TCTGTGTCAC

622 CCACACAAAC CAGTTGCTTT CCAGGAACCT GTTAGATGTA TTACACAGAC AAAAACAGTA

682 CACACAGAGC CCAGAATTCT GATTCCCCCC TCAGATAACT GCTCCAGCCT GTCTACTTTC

742 CCAAGCCAAC TCCTGAAACA CCCACTGTTA CCCAAAGCCC CAGCACGGCC CCTAGACCAG

802 ACTGGTCACT CCCCAACATT TCCTTCACCA GTCCCTATCC GCCCCCACCG AATACTACCT

862 GACTATGAAG AAAAGGCCCA GGAAGGCTTT CAGCCCCAAT AGTGGCACAT AAGTGAGGAA

922 GAAGCGGACG TAGAAGGTAA TCATCCAGGC CAAGTCCTAT AGTGAGAAAA GCAGCGAGCA

982 TAACAGTCAC GAACAACTC

Figure 3. Human partial genomic sequence containing the targeted SNP rs174547 labelled with Y; Primer $\mathrm{F}$ and Primer $\mathrm{R}$ are indicated with bold and underlined.

Table 1. Parameters of the designed primer set with FADS1-F and FADS1-R primer

\begin{tabular}{|l|l|l|l|l|l|}
\hline Primer name & Start point & length & \multicolumn{1}{|c|}{$\mathrm{T}^{0} \mathrm{~m}$} & GC\% content & \multicolumn{1}{|c|}{ 5'-3'sequence $^{\text {'s }}$} \\
\hline FADS1-F & 408 & $20 \mathrm{bp}$ & 59.12 & 55.00 & CACTCCCTTCACATGGTGC \\
\hline FADS1-R & 650 & $20 \mathrm{bp}$ & 59.04 & 55.00 & GGTTCCTGGAAAGCAACTGG \\
\hline
\end{tabular}




\section{RESULTS AND DISCUSSION}

It was shown that under the optimized reaction condition the designed primer system by us produced an amplicon with the expected size (243bp) and contained a fragment from the FADS1 gene, with the analyzed C/T SNP inside.

The results from the agars gel electrophoresis show very discriminative illuminating band as PCR product located between 200 and 300 bp compared with the molecular marker. There are other bands that can be seen on the agar gel between 100 and $200 \mathrm{bp}$, indicating the presence of other PCR product with smaller molecular mass than the targeted one. However, the quality of the aimed product is not compromised, concluding from the strength of the signal from the Chemiluminescence Imaging System.

It was demonstrated that the designed FADS1 pair of primers are specific and reliable tool for obtaining the targeted amplicon. The following sequencing by Sanger revealed the exact nucleotide variation, classifying the unknown samples as homozygote (samples having only allele $\mathrm{C}$ or allele $\mathrm{T}$ ) and heterozygote (samples having both allele $C$ and allele $T$ ). This information gives researchers vision of the magnitude of influence of the given SNP over the metabolic pathways and also has a predictive role for developing future chronic illnesses and conditions. But the science of nutrigenomic and nutrigenetics has definitely optimistic position about that knowledge and says that most of the negative consequences predicted by presence or absence of a particular SNP could be modified and affected by simple changes in the lifestyle and nutritional habits. This gives humanity another, proven by science, line of defense against developing future chronic diseases such as heart disease, type 2 diabetes, obesity, insulin resistance, Alzheimer's disease and other.

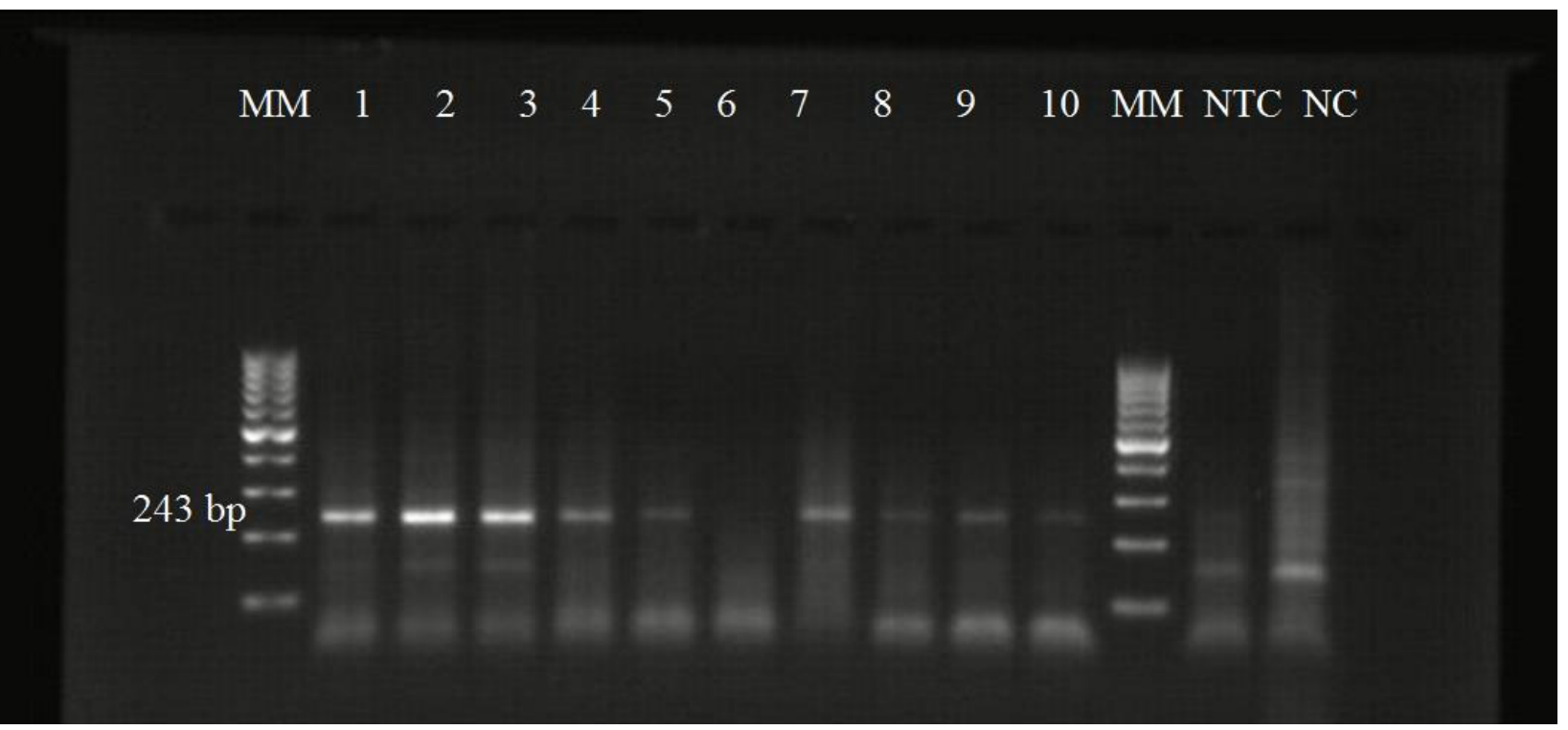

Figure 4. Gel electrophoresis following conventional PCR reaction for visualization of the targeted product. The expected amplicon is $253 \mathrm{bp}$ and positions from 1 to 10 are unknown samples; MMmolecular marker (100 bp DNA Ladder); NTC- non template controls, NC-negative control (bacterial DNA)

\begin{tabular}{|c|c|}
\hline $151 / 454$ & $111 C=$ \\
\hline 2. S1 & 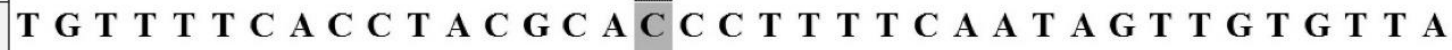 \\
\hline 3. $\mathrm{S2}$ & 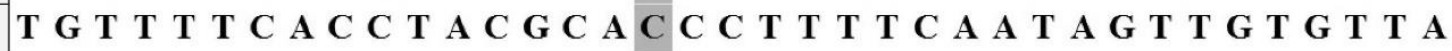 \\
\hline 4. $\mathrm{S3}$ & 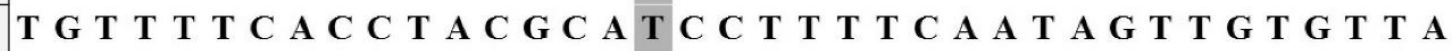 \\
\hline 5. $\mathrm{S4}$ & T T C A C C T A C G C A T C C T T T T C A A T A G T T G T G T T \\
\hline 6. S5 & 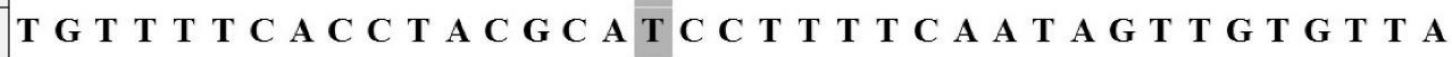 \\
\hline 7. S6 & 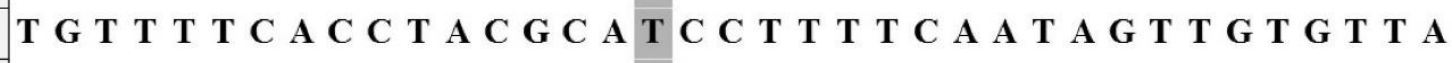 \\
\hline 8. S7 & 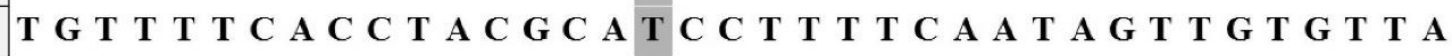 \\
\hline 9. S8 & 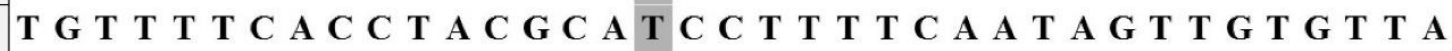 \\
\hline 10. S9 & T T C A C C T A C G C A T C C T T T \\
\hline 11. S10 & T G T T T T C A C C $\mathbf{C}$ T A C G C A $\mathbf{T}$ C C C \\
\hline
\end{tabular}

Figure 5. Results from Sanger DNA Sequencing. The presented figure is part of the consensus alignment. S1 to S10 are the examined samples; rs174547 is human partial genomic sequence obtained from dbSNP genome database. In the alignment sample S1 and S2 are heterozygous (C/T) both containing the $\mathrm{C}$ variant and T allelic variant. 


\section{CONCLUSION}

The proposed system for detection and analysis of SNPs in the FADS1 gene was optimized and probated for the first time in Bulgaria. It has showed its efficacy and reliability for further implementations concerning SNP molecular research in the field of genetics, nutrigenetics and personalized medicine. Thus, although a small-scale pilot study, its results represent primary data originated from Bulgarian individuals. The received results and the collected data may serve as a starting point for future epidemiological and large-scale population studies.

\section{REFERENCES}

1. Schaeffer L, Gohlke H, Muller M, Heid IM, Palmer LJ, Kompauer I, et al. Common genetic variants of the FADS1 FADS2 gene cluster and their reconstructed haplotypes are associated with the fatty acid composition in phospholipids. Human molecular genetics. 2006;15(11):1745-56.

2. Nakamura MT, Nara TY. Structure, function, and dietary regulation of delta6, delta5, and delta9 desaturases. Annu Rev Nutr. 2004;24:345-76.

3. Marquardt A, Stohr $H$, White $K$, Weber $B H$. cDNA cloning, genomic structure, and chromosomal localization of three members of the human fatty acid desaturase family. Genomics. 2000;66(2):175-83.

4. Chasman DI, Pare G, Mora S, Hopewell JC, Peloso G, Clarke R, et al. Forty-three loci associated with plasma lipoprotein size, concentration, and cholesterol content in genome-wide analysis. PLoS genetics. 2009;5(11):e1000730.

5. Xie L, Innis SM. Genetic variants of the FADS1 FADS2 gene cluster are associated with altered $(n-6)$ and $(n-3)$ essential fatty acids in plasma and erythrocyte phospholipids in women during pregnancy and in breast milk during lactation. The Journal of nutrition. 2008;138(11):22228.

6. Merino DM, Ma DW, Mutch DM. Genetic variation in lipid desaturases and its impact on the development of human disease. Lipids Health Dis. 2010;9:63.

7. de Antueno RJ, Knickle LC, Smith H, Elliot ML, Allen SJ, Nwaka S, et al. Activity of human Delta5 and Delta6 desaturases on multiple $n-3$ and $n-6$ polyunsaturated fatty acids. FEBS letters. 2001;509(1):77-80.

8. Moro K, Nagahashi M, Ramanathan R, Takabe $\mathrm{K}$, Wakai T. Resolvins and omega three polyunsaturated fatty acids: Clinical implications in inflammatory diseases and cancer. World J Clin Cases. 2016;4(7):155-64.

9. Zamaria N. Alteration of polyunsaturated fatty acid status and metabolism in health and disease. Reprod Nutr Dev. 2004;44(3):273-82.

10. Wijendran $V$, Hayes KC. Dietary $n-6$ and $n-3$ fatty acid balance and cardiovascular health. Annu Rev Nutr. 2004;24:597-615.

11. Sala-Vila A, Miles EA, Calder PC. Fatty acid composition abnormalities in atopic disease: evidence explored and role in the disease process examined. Clin Exp Allergy. 2008;38(9):1432-
50 .

12. Nakada T, Kwee IL, Ellis WG. Membrane fatty acid composition shows delta-6-desaturase abnormalities in Alzheimer's disease. Neuroreport. $1990 ; 1(2): 153-5$.

13. Dennis EA. Lipid Cell Signaling, Enzymes, LIPID MAPS, and Mediators of Inflammation. J Biol Chem. 2016.

14. Shapiro $H$, Singer $P$, Ariel A. Beyond the classic eicosanoids: Peripherally-acting oxygenated metabolites of polyunsaturated fatty acids mediate pain associated with tissue injury and inflammation. Prostaglandins Leukot Essent Fatty Acids. 2016;111:45-61.

15. Simopoulos AP, Ordovas JM. Nutrigenetics and nutrigenomics. Basel ; New York: S. Karger; 2004. xii, 324 p. p.

16. Park MH, Kim N, Lee JY, Park HY. Genetic loci associated with lipid concentrations and cardiovascular risk factors in the Korean population. Journal of medical genetics. 2011;48(1):10-5.

17. Lanktree MB, Hegele RA. Gene-gene and gene-environment interactions: new insights into the prevention, detection and management of coronary artery disease. Genome Med. 2009;1(2):28.

18. Aulchenko YS, Ripatti S, Lindqvist I, Boomsma D, Heid IM, Pramstaller PP, et al. Loci influencing lipid levels and coronary heart disease risk in 16 European population cohorts. Nature genetics. 2009;41(1):47-55.

19. Kathiresan S, Willer CJ, Peloso GM, Demissie S, Musunuru K, Schadt EE, et al. Common variants at 30 loci contribute to polygenic dyslipidemia. Nature genetics. 2009;41(1):56-65.

20. Teslovich TM, Musunuru K, Smith AV, Edmondson AC, Stylianou IM, Koseki M, et al. Biological, clinical and population relevance of $95 \mathrm{loci}$ for blood lipids. Nature. 2010;466(7307):70713.

21. Dupuis J, Langenberg C, Prokopenko I, Saxena $R$, Soranzo $N$, Jackson $A U$, et al. New genetic loci implicated in fasting glucose homeostasis and their impact on type 2 diabetes risk. Nature genetics. 2010;42(2):105-16

22. Hellstrand $S$, Sonestedt $E$, Ericson $U$, Gullberg B, Wirfalt E, Hedblad B, et al. Intake levels of dietary long-chain PUFAs modify the association between genetic variation in FADS and LDL-C. Journal of lipid research. 2012;53(6):1183-9.

23. Wang $X$. Lipid signaling. Curr Opin Plant Biol. 2004;7(3):329-36.

24. Dinasarapu AR, Saunders B, Ozerlat I, Azam K, Subramaniam $S$. Signaling gateway molecule pages--a data model perspective. Bioinformatics. $2011 ; 27(12): 1736-8$.

25. Lingwood $D$, Simons K. Lipid rafts as a membrane-organizing principle. Science. 2010;327(5961):46-50.

26. Szoor A, Szollosi J, Vereb G. Rafts and the battleships of defense: the multifaceted microdomains for positive and negative signals in immune cells. Immunol Lett. 2010;130(12):2-12. 